\title{
LAS PERCEPCIONES DEL APRENDIZAJE, FENÓMENO INFLUYENTE EN LA FORMACIÓN DOCENTE DE CALIDAD
}

\author{
PERCEPTIONS OF LEARNING, INFLUENCING PHENOMENON IN QUALITY TEACHING \\ TRAINING
}

\author{
Ronald José Feo Mora ${ }^{1}$ \\ feoronald@gmail.com \\ Instituto Pedagógico de Miranda \\ Caracas, Venezuela
}

\author{
Volumen 8, Número 1 \\ 30 de mayo de 2017 \\ pp. $21-53$
}

Recibido: 30 de mayo de 2016

Aprobado: 25 de abril de 2017

1 Ronald José Feo Mora, Instituto Pedagógico de Miranda, José Manuel Siso Martínez, Caracas, Venezuela. Universidad Pedagógica Experimental Libertador de Venezuela. Correo electrónico: feoronald@gmail.com

Las percepciones del aprendizaje, fenómeno influyente en la formación docente de calidad Ronald José Feo Mora

DOI: http://dx.doi.org/10.22458/caes.v8i1.1772

\section{(c) $(7)$ ()}

Artículo protegido por licencia Creative Commons 


\section{Resumen}

En los centros de formación docente emerge un fenómeno influyente y básico en la praxis educativa de calidad, constituido por las percepciones del aprendizaje de sus actores clave. Dicha afirmación impulsa el estudio de este constructo con la finalidad de develar y actualizar los elementos esenciales como distintivos para su conceptualización. Este estudio se insertó en el paradigma naturalístico, donde el método para procesar la información e interpretarla en su esencia fue el fenomenológico. Los informantes iniciales lo conformaron estudiantes y profesores del curso Desarrollo de Procesos Cognoscitivos (DPC 0113) del Instituto Pedagógico de Miranda José Manuel Siso Martínez de Venezuela en los períodos académicos 2015-II - 2016-I. Bajo los principios de la saturación teórica, se determinaron los 12 informantes finales. Las técnicas empleadas para recabar la información fueron la observación participante, apoyada en una guía de observación y la entrevista en profundidad, sustentada en un guion de entrevista. Los hallazgos emergentes permiten afirmar, que la percepción sobre el aprendizaje en la formación docente continuamente se transforma a la par de las acciones cognitivas, afectivas y éticas de los sujetos que la conforman. Este fenómeno permite construir aproximaciones teóricas que permiten generar acciones para una formación docente de calidad.

Palabras clave: Percepciones, Aprendizaje; Formación Docente, Calidad

\section{Summary}

In the centers of teacher training emerges an influential and basic phenomenon in the quality educational praxis, constituted by the perceptions of the learning of its key actors. This affirmation promotes the study of this construct with the purpose of unveiling and updating the essential elements as distinctive for its conceptualization. This study was inserted in the naturalistic paradigm, where the method to process the information and to interpret it in its essence was the phenomenological one. The initial informants were made up of students and professors of the Cognitive Processes Development Course (DPC 0113) of the Miranda Pedagogical Institute José Manuel Siso Martínez de Venezuela in the academic periods 2015-II - 2016-I. Under the principles of theoretical saturation, the 12 final informants were determined. The techniques used to gather information were participant observation, supported by an observation guide and the in-depth interview, based on an interview script. The emerging findings allow us to affirm that the perception about learning in teacher training continually changes along with the cognitive, affective and ethical actions of the subjects that make it up. This phenomenon allows us to construct theoretical approaches that allow us to generate actions for quality teacher training.

Keywords: Perceptions, Learning; Teacher Training, Quality

Las percepciones del aprendizaje, fenómeno influyente en la formación docente de calidad Ronald José Feo Mora

DOI: http://dx.doi.org/10.22458/caes.v8i1.1772

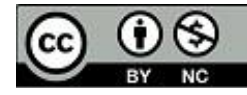

Artículo protegido por licencia Creative Commons 


\section{MANIFESTACIONES PRELIMINARES}

Las exigencias de la sociedad, del desarrollo humano y de la evolución del conocimiento han suscitado diversos cambios que impactan en todas las regiones del mundo. El creciente flujo de información, el llamado a la diversidad concertada, la demanda de competencias específicas, la verificación científica y el tecnicismo ante el compartir de saberes, la globalización de la dominación y la necesaria inclusión social hacen girar la mirada hacia el ser humano, hacia su consciencia; por tanto, los acuerdos construidos en sociedad deben considerar la pluralidad y el acceso al conocimiento.

Desde esta perspectiva de renovación de la formación docente parece profesarse la posibilidad de un mejor futuro en el seno de una sociedad democrática, autónoma, crítica y creativa, en la que los seres humanos liberados de las actitudes de conformismo que imponen los sistemas educativos promotores de la heteronomía y de la visión mercantilista sean capaces de autogestionarse, de decidir por sí mismos y de reflexionar sobre su praxis diaria.

La idea anterior hace considerar que los gobiernos, las universidades junto a los actores clave como lo son docentes y estudiantes, están convocados a actuar de manera conjunta en la búsqueda de acciones coherentes, flexibles, creativas e innovadoras que fortalezcan la formación docente de calidad. En consecuencia, a estas aserciones se presenta un estudio que tuvo como objetivo central analizar las percepciones sobre el aprendizaje que poseen los docentes y los estudiantes en el Instituto Pedagógico de Miranda José Manuel Siso Martínez adscrito a la Universidad Pedagógica Experimental Libertador de Venezuela.

El propósito enunciado pretende sentar orientaciones que permitan construir aproximaciones teóricas que consientan generar acciones para una formación docente pertinente y actualizada, fundamentada en orientaciones hacia la calidad, a la par de comprender, desde

Las percepciones del aprendizaje, fenómeno influyente en la formación docente de calidad Ronald José Feo Mora

DOI: http://dx.doi.org/10.22458/caes.v8i1.1772

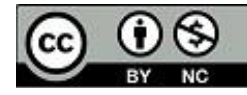

Artículo protegido por licencia Creative Commons 
la realidad de sus actores, la percepción de aprendizaje que facilite propuestas de formación acordes a los elementos esenciales que caracterizan dicho concepto.

\section{FUNDAMENTACIÓN REFERENCIAL}

\section{La Percepción}

En 1986 Abbagnano resalta que la percepción no responde a un fenómeno lineal de estímulos y de respuestas, lo que permite concebir al sujeto como un ente activo tanto mentalmente como socialmente, esto permite afirmar que en la percepción humana intervienen procesos, una actividad mental interactiva e influyente entre el individuo y la sociedad, donde este último hace vida. En el proceso de la percepción están involucrados mecanismos vivenciales que implican el ámbito consciente y el inconsciente de la psique humana, en el que interviene la selección de preferencias, prioridades, diferencias cualitativas y cuantitativas del individuo acerca de lo que percibe.

Para Vargas (1994), la principal disciplina en términos generales que se ha encargado del estudio de la percepción ha sido la psicología, y tradicionalmente este campo ha definido a la percepción como el proceso cognitivo de la consciencia que consiste en el reconocimiento, la interpretación y significación para la elaboración de juicios en torno a las sensaciones obtenidas del ambiente físico y social en el que intervienen otros procesos psíquicos, entre los que se encuentran el aprendizaje, la memoria y la simbolización. No obstante, que la percepción ha sido concebida como un proceso cognitivo, hay autores que la consideran como un proceso más o menos distinto, señalando las dificultades de plantear las diferencias que esta tiene con el proceso del conocimiento.

El mismo autor afirma, que uno de los aspectos de mayor acuerdo entre los estudiosos de la percepción es el de la elaboración de juicios, planteada como una de las características esenciales de este fenómeno humano. Los juicios y su formulación han sido abordados como procesos cognitivos, activados de manera consciente, en un sistema, en donde el sujeto es estimulado, tiene sensaciones y las intelectualiza manifestando juicios u opiniones

Las percepciones del aprendizaje, fenómeno influyente en la formación docente de calidad Ronald José Feo Mora

DOI: http://dx.doi.org/10.22458/caes.v8i1.1772

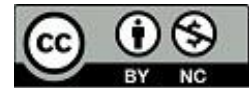

Artículo protegido por licencia Creative Commons 
de las sensaciones y estímulos precisados; estas ideas ciñen a la percepción como un proceso cognitivo de la mente consciente.

En la percepción influyen referentes ideológicos y culturales que representan y explican la realidad y, que en consecuencia se implementan a las experiencias diarias que el sujeto activo ejerce. Esta influencia de los referentes ideológicos y culturales impulsa que las experiencias sean ordenadas, memorizadas y transformadas; durante estos tres procesos emerge el reconocimiento de las experiencias. El reconocimiento permite al ser humano evocar experiencias y conocimientos previamente adquiridos, los cuales pueden ser compartidos con las nuevas experiencias, lo que permite clasificarlas, enriqueciendo las concepciones y definiciones que el entorno exige.

\section{El Aprendizaje Humano}

Hernández (1998), Pozo (1999) y Moya (2007), sustentados en el modelo de procesamiento humano de información, dividen al aprendizaje en cuatro niveles fundamentales: (a) la conducta; (b) las capacidades; (c) las habilidades; y (d) las competencias. La conducta y las capacidades consolidan un aprendizaje verbal y conceptual, "saber qué", representado cuando el sujeto adquiere información sobre hechos y datos. El sujeto que aprende debe hacer una copia más o menos literal o exacta de la información proporcionada y almacenarla en su memoria. Este carácter reproductivo del aprendizaje de datos y hechos hace que el proceso fundamental sea la repetición o repaso de los materiales de aprendizaje.

El que aprende para procesar información se apoya en técnicas que le permiten memorizar el contenido de estudio. Las habilidades aseguran un aprendizaje de procedimientos, "saber cómo", representado a través de la adquisición de técnicas, construcción de estrategias de aprendizaje, orientadas a la consecución de una meta. Los procesos cognitivos son esenciales para la comprensión de los procedimientos, los cuales son usados para la adquisición y construcción de conocimientos de manera activa y significativa. Para que el

Las percepciones del aprendizaje, fenómeno influyente en la formación docente de calidad Ronald José Feo Mora

DOI: http://dx.doi.org/10.22458/caes.v8i1.1772

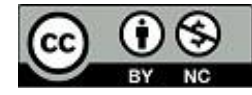

Artículo protegido por licencia Creative Commons 
sujeto desarrolle habilidades debe seguir instrucciones, practicar los procedimientos y aplicarlos de manera pertinente. Los afectos y las motivaciones representan un eje medular del aprendizaje.

Las competencias son sistemas complejos que integran las capacidades, las habilidades, los valores y la ética en un sujeto. Las competencias le permiten al individuo movilizar dicha integración como recursos para resolver una tarea; posee un buen desempeño, todo sujeto que emplea sus competencias de manera eficiente en correspondencia a las exigencias del contexto donde se desarrolla su actividad. El logro y desarrollo de competencias está asociado con el aprendizaje de habilidades sociales, estas se producen en contextos donde existe la interacción social que determina en buena medida la dirección y el significado de lo que se aprende. El sujeto que aprende para procesar información se apoya en técnicas de estudio, en estrategias de aprendizaje, en el intercambio de saberes con sus pares y en sus motivos.

La comprensión del aprendizaje y los procesos influyentes sobre él, es, sin duda, uno de los temas relevantes de las comunidades científicas relacionadas a los sistemas formales de educación; por esta razón, las representaciones del aprendizaje humano siguen y seguirán en una constante construcción, esto significa que mientras evoluciona el ser humano y junto a él la tecnología que facilita su vida, la aproximación a la comprensión del aprendizaje poseerá mayor veracidad y credibilidad, pero sobre todo, una mayor apropiación de significado humano que le precede.

Así pues, desde el inicio de la humanidad el hombre ha sentido la necesidad de ajustar y preservar sus saberes en consonancia a un contexto que lo demanda y así trascender. Ese sin duda es el comienzo de la enseñanza, caracterizado por procesos afectivos y cognitivos que evolucionaron a la par del mismo individuo, su conciencia y sus valores. Es así, como en la búsqueda de procesos pedagógicos de mayor eficiencia y flexibilidad surgió la percepción del aprendizaje y con él un vuelco en la historia de la humanidad; esto permitió

Las percepciones del aprendizaje, fenómeno influyente en la formación docente de calidad Ronald José Feo Mora

DOI: http://dx.doi.org/10.22458/caes.v8i1.1772

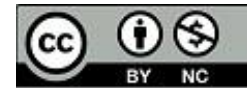

Artículo protegido por licencia Creative Commons 
centrar la mirada sobre el ente de aprendizaje y no en exclusivo sobre el ente de enseñanza.

La construcción del aprendizaje se realiza todos los días y en casi todos los contextos de la vida, y depende de la representación inicial que se tiene de la nueva información y de la actividad externa o interna que se desarrolla al respecto. Todo aprendizaje supone una construcción que se realiza a través de un proceso mental que conlleva a la adquisición de un conocimiento nuevo, o a reafirmar lo ya conocido; además, la posibilidad de construirlo y lograr una nueva competencia, que le permitirá a la persona transferir lo ya conocido a una nueva situación.

Finalmente, la definición del concepto de aprendizaje, basándose en los enunciados sobre percepción y aprendizaje antes expuestos, se reconoce como un proceso constructivo y autoestructurante de saberes, que le permite al sujeto la organización interna de información en esquemas mentales, y estos le proporcionan la solución al conflicto que emerge entre lo que él sabe y lo que desea saber, facilitado a través de la interacción con sus pares y las estrategias de aprendizaje que enriquecen la comprensión de información. El aprendizaje debe ser visto como un fenómeno psicológico, afectivo, social.

\section{La Formación Docente}

Cada modelo de formación docente, para De Lella (1999), articula concepciones acerca de educación, de enseñanza, de aprendizaje y las recíprocas interacciones que las afectan o determinan, permitiendo una visión totalizadora del sujeto. Los distintos modelos, hegemónicos en un determinado momento histórico, no configuran instancias monolíticas o puras, dado que se dan en su interior contradicciones y divergencias; ellas mismas coexisten, influyéndose bilateralmente. La historia de los programas de formación docente, específicamente en la formación inicial, con énfasis en sus prácticas, definitivamente, han tenido una función significativa y definitoria de los modelos y tendencias en la formación docente; Sánchez y Jurado (2000) lo explican de la siguiente manera:

Las percepciones del aprendizaje, fenómeno influyente en la formación docente de calidad Ronald José Feo Mora 
Por un lado, las prácticas son asumidas como el desarrollo de una serie de asignaturas donde los estudiantes y futuros educadores deben cumplir. Estas asignaturas tienen dos maneras de caracterizarse; la primera, es vista como ejercicio en el salón de clases bajo la guía de un plan previo diseñado y la segunda, según las orientaciones del profesor / supervisor de la práctica y con lo cual el futuro profesor demuestra competencia para la docencia. Por el otro, se asume la práctica en la perspectiva de la investigación, donde el estudiante fundamenta un proyecto articulado en una línea de investigación en el plan de estudios, a su vez puede ser combinada con intercambios y trabajos en la comunidad a través del desarrollo de un proyecto conjunto al centro de formación (p. 55).

Estas dos tendencias suponen concepciones distintas del aprendizaje y de los desempeños del estudiante y del profesor; la primera tendencia da cuenta de una concepción conductista del aprendizaje, que lo visualiza como un ente pasivo, el cual desarrolla habilidades y destrezas que recibe conocimientos teóricos y didácticos para luego aplicarlos a la realidad; la segunda tendencia concibe el aprendizaje como una construcción social a partir de situaciones y actividades contextualizadas y situadas; el desempeño del estudiante es activo y constructor de sus aprendizajes en interacción con sus pares y con los otros mediadores sociales.

En correspondencia a lo descrito y con base en De Lella (1999) y Tejada (2000) sobre la descripción, la clasificación de los modelos y las tendencias, configurados históricamente e incorporadas a las prácticas docentes y a la conciencia de los sujetos, se puede puntualizar lo siguiente:

El modelo práctico - artesanal concibe a la enseñanza como una actividad artesanal, un oficio que se aprende en el taller. El conocimiento profesional se transmite de generación en generación y es el producto de un largo proceso de adaptación a la escuela y a su función de socialización. El aprendizaje del conocimiento profesional supone un proceso de inmersión en la cultura de la escuela mediante el cual el futuro docente se socializa dentro de la institución, acepta la cultura profesional heredada y los roles profesionales correspondientes.

El modelo academicista específica que lo esencial de un docente es su sólido conocimiento de la disciplina que enseña. La formación así llamada pedagógica, como si no fuera también disciplinaria, pasa a un segundo plano y suele considerarse superficial y hasta innecesaria.

Las percepciones del aprendizaje, fenómeno influyente en la formación docente de calidad Ronald José Feo Mora 
Los conocimientos pedagógicos podrían conseguirse en la experiencia directa en la escuela, dado que cualquier sujeto con buena formación conseguiría orientar la enseñanza, esto genera una brecha entre producción y reproducción del saber. El docente no necesita el conocimiento experto, sino las competencias requeridas para trasmitir el guion elaborado por la comunidad de expertos.

El modelo técnicista eficientista apunta a tecnificar la enseñanza, sobre la base de esta racionalidad, con economía de esfuerzos y eficiencia en el proceso y los productos. El docente es esencialmente un técnico, su labor consiste en bajar a la práctica, de manera simplificada, un currículo prescrito por expertos externos en torno a objetivos de conducta y medición de rendimientos. El docente no necesita dominar la lógica del cocimiento científico, sino las técnicas de transmisión, está subordinado, no solo al científico de la disciplina, sino también al pedagogo y al psicólogo.

El modelo hermenéutico reflexivo supone a la enseñanza como una actividad compleja, en un ecosistema inestable, sobre determinada por el contexto y espacio temporal, sociopolítico y a la vez cargado de conflictos de valor que requieren opciones éticas y políticas. El docente conjuntamente con sus estudiantes debe enfrentar, con sabiduría y con creatividad, situaciones en prácticas imprevisibles que exigen a menudo resoluciones inmediatas para las que no sirven reglas técnicas ni recetas de la cultura escolar. Vincula lo emocional con la indagación teórica, se construye personal y colectivamente, parte de situaciones concretas que intenta reflexionar y comprender con herramientas conceptuales y vuelve a la práctica para modificarla. Posiblemente la vigencia del modelo hermenéutico reflexivo se evidencia con mayor contundencia en la praxis cotidiana de los centros de formación, más que en los currículos propios de la formación docente; es decir, se evidencia en la interacción social emergente entre los sujetos que comparten experiencias de enseñanza y de aprendizaje.

Al analizar la presencia de los modelos y tendencias de la formación docente es evidente que gran parte de sus programas, a excepción del modelo hermenéutico reflexivo, fueron influenciados por modelos y tendencias que promocionan la conducción de los aprendizajes, donde el desempeño

Las percepciones del aprendizaje, fenómeno influyente en la formación docente de calidad Ronald José Feo Mora

DOI: http://dx.doi.org/10.22458/caes.v8i1.1772

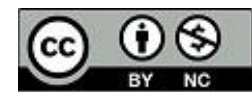

Artículo protegido por licencia Creative Commons 
docente y del estudiante es notoriamente pasivo, trasmisor y receptor de información, de la cultura, de las certezas de los últimos descubrimientos científicos, de la racionalidad técnica. Situación que acrecienta la posibilidad de formar a un sujeto no crítico del entorno, orientado al seguimiento de conductas sociales, alejado a la comprensión de las mismas. Sin embargo, la actualidad del siglo XXI demanda otro tipo de docente, otro tipo de estudiante; en otras palabras, otro profesional de la docencia, el cual no declare contenidos exclusivamente, pero que domine los contenidos de su área de acción a la par de los elementos pedagógicos para su declaración, centrados en el que aprende y no en el que enseña.

Es necesario un docente que promueva procesos e impulse al desarrollo de habilidades para comprender las situaciones problemáticas, y a su vez impulse los valores sociales necesarios para formar a un sujeto integral, ya que el contexto así lo demanda. En definitiva, un docente con voluntad para el progreso que perciba que estar actualizado en sus competencias profesionales es una prioridad.

Estas aserciones reiteran que la transmisión de conocimientos de saberes disciplinares es una visión arcaica de la formación docente; hoy se abre paso a la construcción de conocimientos, el procesamiento de la información colectiva que impacta en la conceptualización de mundo individual, en la inclusión y en el respeto al otro. No se trata de encubrir al estudiante, se trata de formarlo en la prosperidad, la voluntad, la responsabilidad, el diálogo y el trabajo, de esta manera se desarrollan individuos con alto nivel cognitivo, procedimental y afectivo.

\section{La Formación Docente de Calidad}

La palabra calidad proviene del latín qualitas que equivale al poites griego, que responde a lo cualitativo o qualitativus en latín, lo que hace referencia a la descripción del ente y su clase. La calidad se orienta al análisis de una propiedad existente o a la comparación con otra similar, afín o de la misma especie. Este aserto concuerda con la definición propuesta por la Subsecretaria de

Las percepciones del aprendizaje, fenómeno influyente en la formación docente de calidad Ronald José Feo Mora

DOI: http://dx.doi.org/10.22458/caes.v8i1.1772

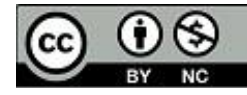

Artículo protegido por licencia Creative Commons 
Educación Superior de México (SESM, 2010) como un conjunto de cualidades positivas deseables de personas, objetos o sistemas.

En la educación también se habla de calidad, la cual está regida por múltiples factores que coexisten en los procesos de enseñanza y aprendizaje, ellos son influenciados por las exigencias del contexto que requieren mayor inclusión, discusión de saberes sobre los conocimientos disciplinares y sobre todo la participación activa del estudiante y la actualización continua del docente; en este mismo sentido, Téllez y otros (2009) orientan la calidad de la educación hacia la presencia del docente, la cual afirman es obligatoria, en su intermediación está la posibilidad de éxito o de fracaso de la innovación de los procesos de enseñanza y aprendizaje, su posición es principal, aunque no suficiente, para garantizar la calidad.

Los mismos autores reafirman, que, si la formación docente es vista como uno de los factores claves hacia el logro de una educación de calidad, se elevaría el nivel de la educación formal y no formal; de esta manera, se alcanzaría a fortalecer los programas de formación docente. Esta necesidad no es una aspiración difusa ni remota, sino factible y urgente. Esto implica, preparar docentes estratégicos, alertas ante las necesidades de las mayorías, conscientes de sus responsabilidades sociales y capaces de impulsar una enseñanza democrática y estimuladora de la investigación, la criticidad, la creatividad y el compromiso solidario para la construcción de un sistema de formación que enseñe para la vida y no en exclusiva para el trabajo.

Bajo estas premisas es necesario reflexionar sobre los sistemas educativos de calidad, sus características que permiten formar a un estudiante de manera integral, que desarrolle sus capacidades humanas a plenitud. Para el Instituto de Ciencias del Hombre la calidad educativa (ICH, 2010) es un concepto multidimensional, constituido por diversos aspectos, que la definen, ellos son:

Calidad como Excepción, poseen dos visiones; la primera, es la clásica donde resalta la distinción, la clase alta y la exclusividad, la segunda, es la actual caracterizada por la

Las percepciones del aprendizaje, fenómeno influyente en la formación docente de calidad Ronald José Feo Mora

DOI: http://dx.doi.org/10.22458/caes.v8i1.1772

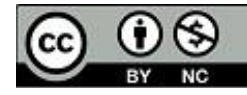

Artículo protegido por licencia Creative Commons 
excelencia, en relación a los estándares, la reputación de los centros, de los recursos y medios y sobre todo por criterios científicos que declaran a los "centros que obtienen buenos resultados".

Calidad como perfeccionamiento o Mérito, la calidad es vista como consistencia de las cosas bien hechas, es decir, que corresponden a los requisitos exigidos; "centros donde las cosas se hacen bien" y promueven la cultura de calidad para que sus resultados sean cada vez mejor evaluados de acuerdo con criterios de control de calidad.

Calidad como adecuación a propósitos, esta tendencia parte de una definición funcional sobre calidad, lo que es bueno o adecuado para algo o alguien. Un ejemplo de esto son los centros donde existe una adecuación entre los resultados, los fines u objetivos propuestos: Los programas y servicios responden a las necesidades de los estudiantes.

Calidad como producto económico, se basa desde la perspectiva del costo que supone su obtención; es decir, centros eficientes al relacionar costos y resultados.

Calidad como transformación y cambio, se centra en la evaluación y la mejora a nivel institucional, caracterizada por centros preocupados por el desempeño del estudiante y porincrementar el valor añadido, orientado hacia el desarrollo cualitativo de la organización "desarrollo organizacional".

Bajo esta descripción, que deja ver las diferentes características y aplicaciones referentes a calidad educativa, es necesario indicar que la más contextualizada, es aquella que centra su visión sobre el desempeño del estudiante y del docente, en el trabajo en equipo, en la comunidad, en la integración teoríca y la práctica. La calidad educativa que demanda el siglo XXI centra sus esfuerzos en el desarrollo de las diversas dimensiones humanas y en la integración del saber - hacer, el saber obrar el saber - pensar.

Las percepciones del aprendizaje, fenómeno influyente en la formación docente de calidad Ronald José Feo Mora

DOI: http://dx.doi.org/10.22458/caes.v8i1.1772

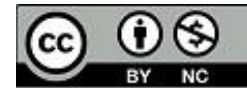


Entonces, por calidad de la educación, se conciben todas aquellas acciones y cualidades que poseen los recursos materiales y los talentos humanos involucrados en los procesos de enseñanza y aprendizaje, que permiten el bienestar en conjunto de cada miembro integrante y el logro común e individual de metas. Cabe resaltar que la calidad de la educación depende en muchos casos de una percepción subjetiva, social o cultural. También, es necesario recalcar que para una formación de calidad, el docente en formación debe tomar conciencia de su entorno, de sus debilidades y fortalezas, comprender que los conocimientos no son aislados, que se construyen en colectivo, que para ser crítico primero hay que ser reflexivo. Estas premisas apuntan hacia la urgente incorporación de acciones pedagógicas y didácticas que formen al docente estratégico, que reconozca las bondades de aprender permanentemente; es decir, que actualice sus competencias constantemente al ritmo de los cambios del contexto.

Afirma Domínguez (2010), que en la calidad de la educación existen múltiples factores; sin embargo, el cumplimiento eficiente de la tarea que la sociedad ha asignado al docente, es un rasgo de elevada relevancia para el desarrollo bio - psico - social del estudiante a su cargo. Las demandas sobre el trabajo docente se acompañan de procesos sistémicos e integrales que le permitan construir nuevas formas de accionar, participar en los cambios y corresponsabilizarse de los resultados de su trabajo.

Para Ponce y otros (2010), la calidad de la educación es un reto para cualquier nación, pues en base a ella se obtienen derivaciones favorables para la sociedad. Las instituciones formadoras de docentes, deben apuntar sus objetivos a ofrecer procesos formativos adecuados a los cambios del mundo, el docente en formación toma gran importancia en este proceso, pues es él quien hará posible la complementación del acto educativo junto a sus estudiantes, por lo que debe estar formado en las competencias necesarias para ejercer su labor de manera adecuada. En correspondencia con lo que se ha descrito sobre la formación docente, se sume en la presente construcción teórica lo siguiente:

1.- Necesidad de un accionar pedagógico y didáctico para la formación docente, centrado en el desarrollo de manera activa e interestructurada a partir del diálogo pedagógico entre el estudiante, el saber y el docente.

Las percepciones del aprendizaje, fenómeno influyente en la formación docente de calidad Ronald José Feo Mora 
2.- Importancia de un accionar pedagógico y didáctico que reconstruya la unidad y la complejidad desde la propia experiencia del que aprende, junto a sus implicaciones emocionales, intelectuales, sociales y éticas.

3.- Llamado a la construcción de una formación docente de calidad que promueva en el sujeto la toma de conciencia, que para ser crítico sobre lo que se aprende, primero hay que ser reflexivo y que los conocimientos no son aislados, se labran en colectivo.

\section{METÓDICA}

Para definir el diseño de una investigación es importante describir las características donde se enmarca su fundamentación metódica, ya que éste influye en la operatividad y el procesamiento de los hallazgos. En este sentido, la investigación estuvo enmarcada en un enfoque interpretativo; esto originó una serie de procedimientos cognitivos y acciones por parte del investigador para describir e interpretar desde varias dimensiones al fenómeno objeto de estudio, lo que permitió contextualizar el método en la investigación.

Taylor y Bogdan (1997) definen a la metodología cualitativa como una forma de entender los fenómenos sociales desde la propia perspectiva del actor, donde la realidad que importa es lo que las personas perciben como trascendental; esto es, comprensión en un nivel personal de los motivos y las creencias que están detrás de los informantes clave. En referencia a las características de la metodología cualitativa explicadas, se asumió para este estudio un diseño abierto, emergente, sistémico, flexible y hasta cierto punto provisional.

De manera específica, el contexto del estudio fue el Instituto Pedagógico de Miranda José Manuel Siso Martínez. Los informantes clave lo conformaron estudiantes y profesores del curso Desarrollo de Procesos Cognoscitivos (DPC 0113) del Instituto Pedagógico de Miranda José Manuel Siso Martínez, períodos 2015-II - 2016-I, siendo 12 actores asumidos como clave. Se describió e interpretó las premisas esenciales encontradas a partir de la comparación entre las

Las percepciones del aprendizaje, fenómeno influyente en la formación docente de calidad Ronald José Feo Mora 
entrevistas en profundidad y las observaciones participantes, lo que permitió emerger categorías propias que definen la percepción del aprendizaje desde el juicio de los estudiantes y docentes.

En este estudio los planteamientos de Husserl (1949) fueron asumidos como referencia filosófica fundamental, para aplicar el método fenomenológico en tres planos de reducción: (a) la reducción fenomenológica, (b) la horizontalización, y (c) la reducción trascendental. Estos planos de reducción permitieron que el fenómeno a ser interpretado pueda explicarse en términos de un sistema intrínseco de significados y no como un dictamen objetivo puesto desde fuera. A continuación, se muestra una figura que describe la contextualización del método fenomenológico en la investigación:

\section{Figura 1. Planos de Reducción Contextualizados en el Estudio.}

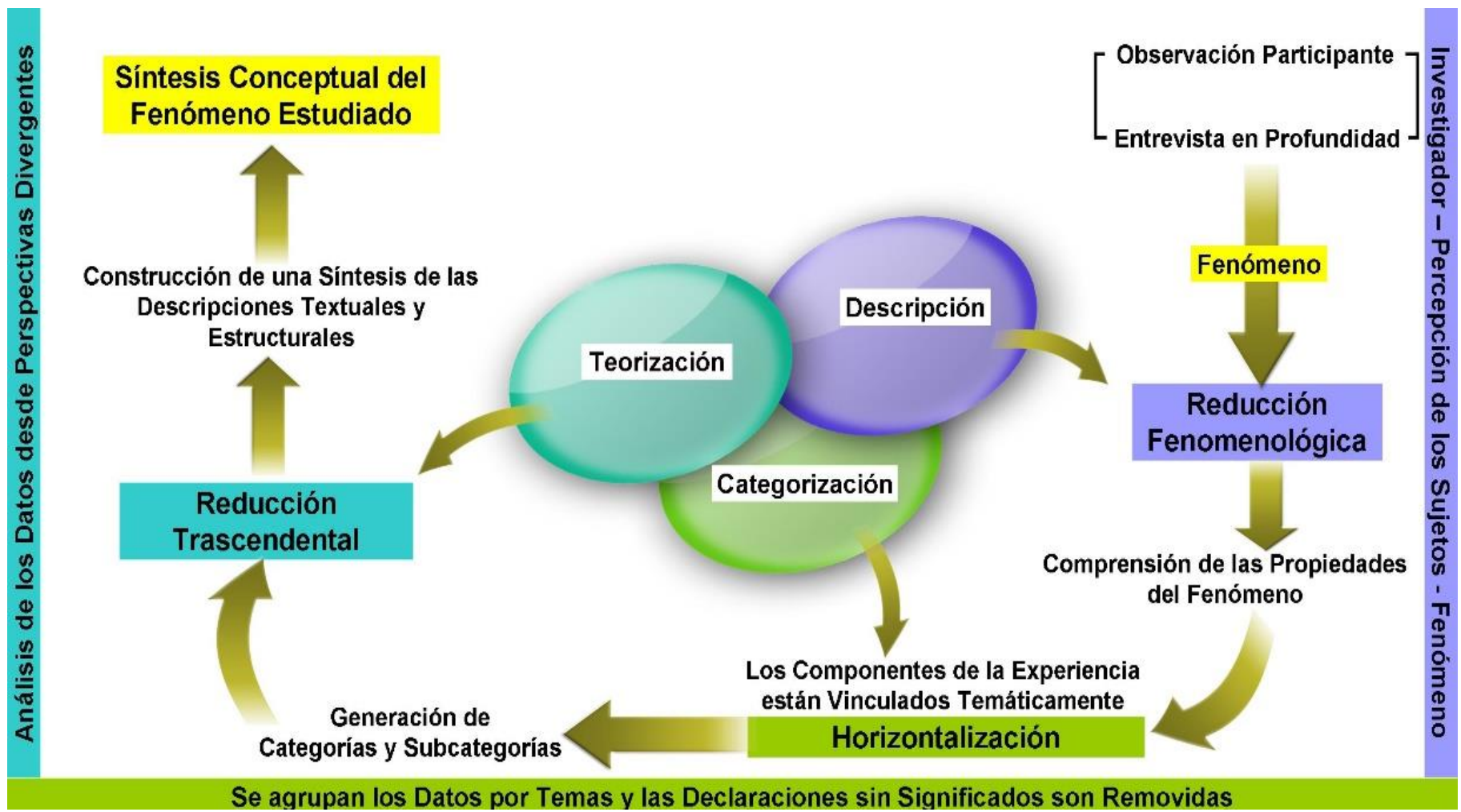

Las percepciones del aprendizaje, fenómeno influyente en la formación docente de calidad Ronald José Feo Mora 
El método fenomenológico para esta investigación tuvo el propósito de permitir, que el mundo de las experiencias de los informantes clave surgiera con claridad para acceder al análisis e interpretación de lo relacionado con la percepción del aprendizaje en la formación docente desde sus actores clave.

A partir de la interrogante emergente durante el estudio, que enunciaba: ¿Cuál es la situación referente a la percepción del aprendizaje en el Instituto Pedagógico de Miranda José Manuel Siso Martínez? La cuál derivó en el objetivo general de la investigación que procuró analizar las percepciones del aprendizaje que poseen los docentes y los estudiantes en el Instituto Pedagógico de Miranda José Manuel Siso Martínez, se realizaron las entrevistas con base en las siguientes preguntas directrices: (a) Explica cómo percibes el aprendizaje; (b) Describe la importancia que tiene el aprendizaje para ti; y (c) Narra lo que haces para aprender. Finalmente, las observaciones participantes se realizaron a partir de las aserciones asumidas en la teoría referencial sobre las percepciones, el aprendizaje y la formación docente. Se realizaron notas de campo a partir de observaciones en los encuentros pedagógicos del curso Desarrollo de Procesos Cognitivos en los períodos académicos 2015-II - 2016-I.

\section{INTERPRETATIVO DE LOS HALLAZGOS}

El interpretativo de los hallazgos en esta investigación es una síntesis descriptiva y coherente de los procedimientos que realizó el investigador para procesar la información recabada a los informantes clave que hacen vida en el escenario donde se implementó el estudio. Igualmente, describe en profundidad la construcción teórica que se alcanzó a través del miramiento de la realidad bajo un enfoque interpretativo.

Las acciones descritas pertenecen a la estrategia investigativa, orientada por el método fenomenológico y sus planos de reducción: (a) fenomenológica; (b) horizontalización; y (c) trascendental, que permitieron caracterizar, analizar e interpretar la información recabada; por consiguiente, comprender la esencia del fenómeno emergente entre la concreción de los constructos: (a) percepción del aprendizaje; y (b) formación docente de calidad.

Las percepciones del aprendizaje, fenómeno influyente en la formación docente de calidad Ronald José Feo Mora 
De la comprensión del fenómeno emergente y su esencia, simultáneamente con los referentes teóricos, se generó una aproximación representativa del objeto de estudio que se nombró síntesis conceptual, la cual permitió develar la esencia del fenómeno. Esta sección del artículo enseña los hallazgos alcanzados durante el desarrollo de la investigación, los cuales fueron fragmentados para una mejor ilustración, en tres planos de reducción que narran o muestran la información procesada y las interpretaciones construidas en cada uno de ellos; los planos fueron: (a) fenomenológica; (b) horizontalización; (b) trascendental. A continuación, se muestran cuadros y gráficos que detallan la sistematización del procesamiento de la información a través de los planos de reducción fenomenológicos asumidos como un ejemplo de lo realizado en profundidad durante la investigación:

\section{Reducción fenomenológica:}

Cuadro 1. Comparación de notas de campo/ entrevista en profundidad (ejemplo de procesamiento)

\begin{tabular}{|c|c|c|c|}
\hline NOTAS DE CAMPO & $\begin{array}{ll}\text { ENTREVISTA } & \text { EN } \\
\text { PROFUNDIDAD } & \end{array}$ & CATEGORÍAS & SUBCATEGORÍAS \\
\hline $\begin{array}{l}\text { 5/10/2015 8: } 00 \text { a.m. a } \\
\text { 12:00 m. } \\
\text { Inf es un estudiante de } \\
\text { sexo masculino de } 19 \\
\text { años de edad, estudia } \\
\text { Física cohorte } 2010 \text {. } \\
\text { Posee estatura media } \\
\text { entre } 1.65 \text { y } 1.70 \mathrm{~cm} \text {., } \\
\text { posee como costumbre } \\
\text { sentarse en el medio } \\
\text { del salón. Casi siempre } \\
\text { interviene, no para de } \\
\text { hablar o preguntar a } \\
\text { sus compañeros o } \\
\text { profesor. Durante la } \\
\text { clase introductoria o de } \\
\text { presentación del curso, } \\
\text { este sujeto expresó } \\
\text { que él venía con la }\end{array}$ & $\begin{array}{l}\text { ¿El aprendizaje? [...] } \\
\text { para mí, se resume en la } \\
\text { capacidad que tenemos, } \\
\text { cada uno de nosotros, } \\
\text { para adquirir [...] asimilar } \\
\text { y procesar cualquier } \\
\text { información } r \text { que } \\
\text { percibimos de nuestro } \\
\text { entorno, y que } \\
\text { estudiamos para luego } \\
\text { aplicarlo e ir adquiriendo } \\
\text { experiencias, y por lo } \\
\text { tanto sumarlo al logro de } \\
\text { nuevos aprendizajes. } \\
\text { (Inf, 02 -05) } \\
\text { Nos permite modificar } \\
\text { nuestra conducta, } \\
\text { desechando lo que no es }\end{array}$ & Aprendizaje & Aprender \\
\hline
\end{tabular}

Las percepciones del aprendizaje, fenómeno influyente en la formación docente de calidad Ronald José Feo Mora 
curiosidad de conocer en realidad que es el aprendizaje, ya que un compañero le dijo que aquí podía conseguirlo. Él ya cursó psicología de la Educación, pero eso no le quedó muy claro, ya que ha leído en otros cursos como, por ejemplo, en Filosofía que el aprendizaje es una capacidad de los seres humanos que le permite adquirir, procesar y asimilar información nueva.

12/10/2015 8: 00 a.m. a 10:00 a.m.

Inf durante la clase de los procesos cognitivos básicos, afirma que con los procesos cognitivos básicos él puede adquirir y comprender la información del entorno, la cultura, el idioma, los valores y las normas impuestas en la sociedad, Sin los procesos

cognoscitivos, aprendizaje no se diera.

12/10/2015 8: 00 a.m. a 10:00 a.m.

Inf durante una presentación de un producto construido en una mesa de trabajo afirmó, como adecuado para nosotros mismos y nos ayuda en el proceso de integración a la sociedad [...] porque tenemos mayor conocimiento sobre nuestra cultura [...] nuestro idioma [...] los valores y normas aceptables dentro de ésta. (Inf, 34 -37)

El aprendizaje es un proceso indispensable en el avance continuo del ser humano, sin este proceso seríamos seres inertes y nos embarcaríamos en la autodestrucción. (Inf, 46 48)

A través del aprendizaje [...] se puedn obtener nuevas habilidades y destrezas para progresar y crecer en cualquier meta que deseamos alcanzar. Tenemos la capacidad de elegir nuestras propias creencias, gustos y prejuicios, importantes en la formación y establecimiento de nuestra personalidad y de cómo de alguna forma empezamos a crear nuestra propia perspectiva del mundo que nos rodea. (Inf, 28 34) conclusión del grupo y

Las percepciones del aprendizaje, fenómeno influyente en la formación docente de calidad Ronald José Feo Mora 


\begin{tabular}{|c|c|c|}
\hline 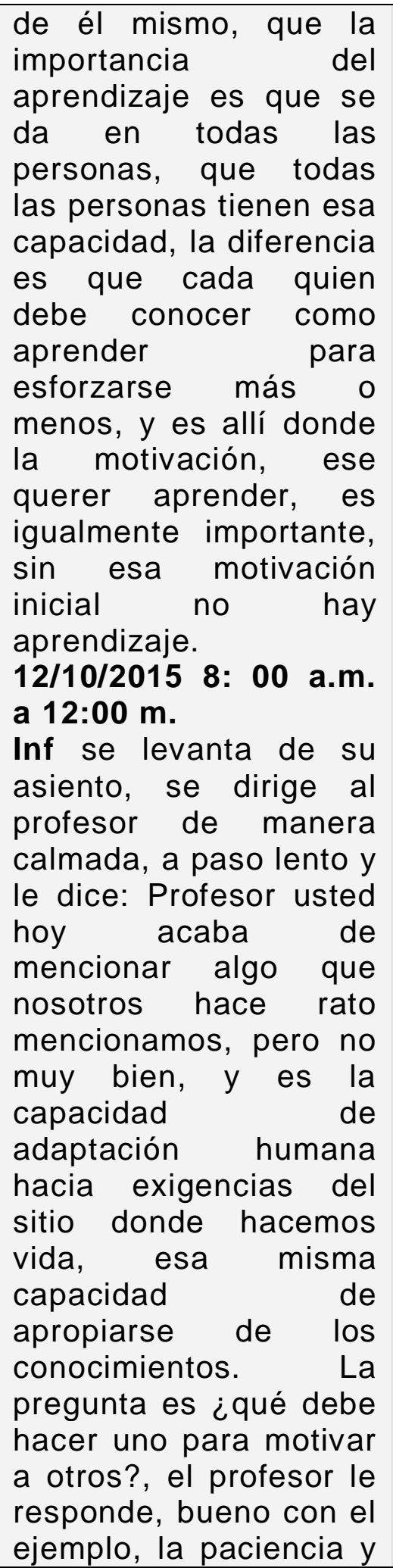 & 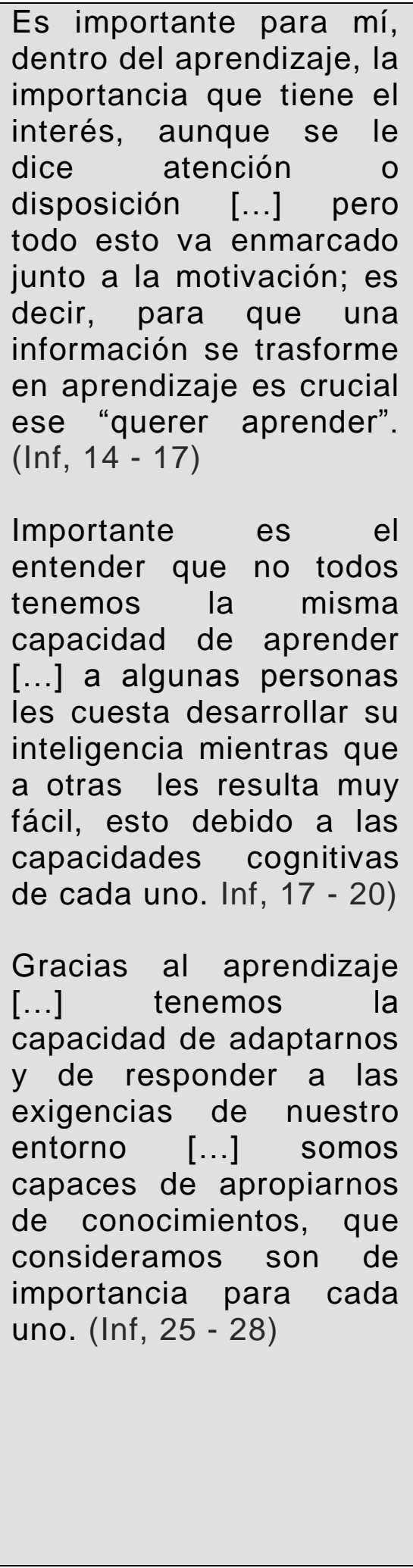 & $\begin{array}{l}\text { Importancia } \\
\text { Aprender }\end{array}$ \\
\hline
\end{tabular}

Las percepciones del aprendizaje, fenómeno influyente en la formación docente de calidad Ronald José Feo Mora 


el estudio, son tres
cosas que un buen
profesor debe hacer
para llamar a otros al
aprendizaje.

Comentarios: Ningún ser humano puede afirmar que ha aprendido algo sin ningún motivo, exigencia o necesidad de aprenderlo. Con el tiempo emerge el reconocimiento de los motivos y, por ende, del aprendizaje que este ha impulsado hasta su logro.

\section{(c) $(1)(8)$}




\section{Horizontalización (ejemplo de procesamiento):}

Cuadro № 2. Categoría de análisis: Aprendizaje

Subcategoría: Aprender

\begin{tabular}{lll}
\hline \hline INTERPRETACIÓN DE & INTERPRETACION & CONTEXTO \\
LOS INFORMANTES & DEL INVESTIGADOR & TEÓRICO \\
\hline \hline
\end{tabular}

El aprendizaje es una capacidad $[\ldots]$

El aprendizaje permite adquirir, asimilar $y$ procesar información

EI aprendizaje modifica las conductas adquiridas $[\ldots]$

El aprendizaje es un proceso que nos ayuda a integrarnos a la sociedad

La dinámica del aprendizaje permite nos ser productivos, activos y sobre todo humanos [...]

Todo ser humano posee la capacidad de aprender, claro a su medida y a sus intereses $[\ldots]$
El aprendizaje es un proceso complejo mediante el cual se utilizan variados recursos para procesar la información y comprenderla,

mientras que aprender es la capacidad humana de generar conceptos, derivados del procesamiento de la información, los cuales son empleados para solventar situaciones

problemáticas. En el proceso de aprender la percepción del sujeto es influyente, ya que no todos los conceptos y sus características esenciales

son

percibidas

semejante de entre los mismo contexto, pero lo que sí es análogo son las características coincidentes que forman el concepto, producto de la mediación y el

Para Flores (2008), la clave en los procesos de enseñanza y aprendizaje está en asegurarse de que el aprendizaje sea significativo, es decir, si el nuevo material de aprendizaje se relaciona de manera sustantiva con lo que el estudiante ya sabe, si es asimilado a su estructura cognitiva, se estará en presencia de un aprendizaje significativo; si, por lo contrario, el estudiante se limita a memorizarlo, sin establecer relaciones con sus conocimientos previos, ocurrirá un aprendizaje repetitivo, memorístico mecánico. Así pues, lo realmente importante es que el

Las percepciones del aprendizaje, fenómeno influyente en la formación docente de calidad Ronald José Feo Mora 


\begin{tabular}{|c|c|}
\hline 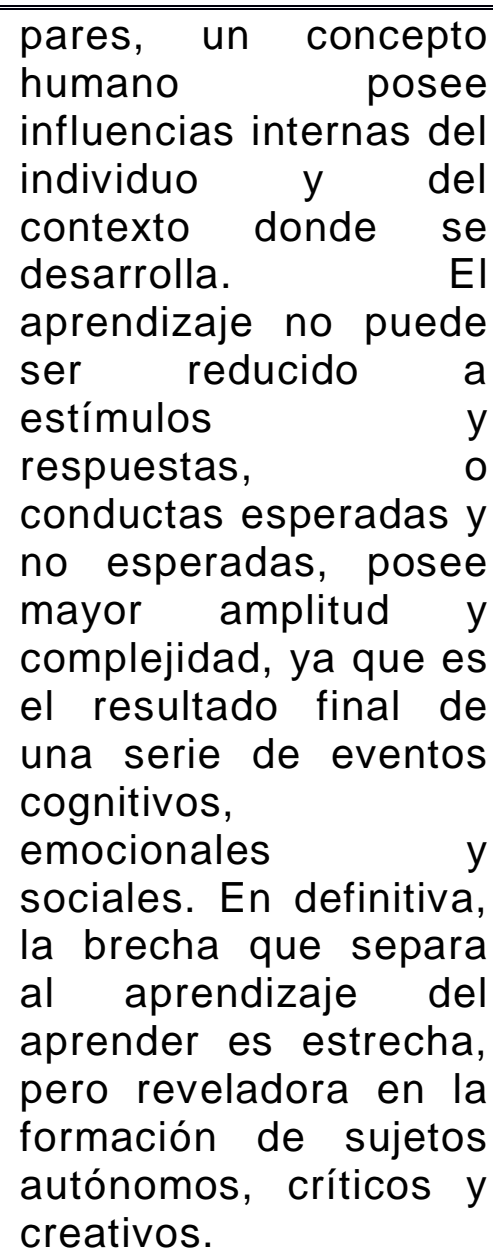 & 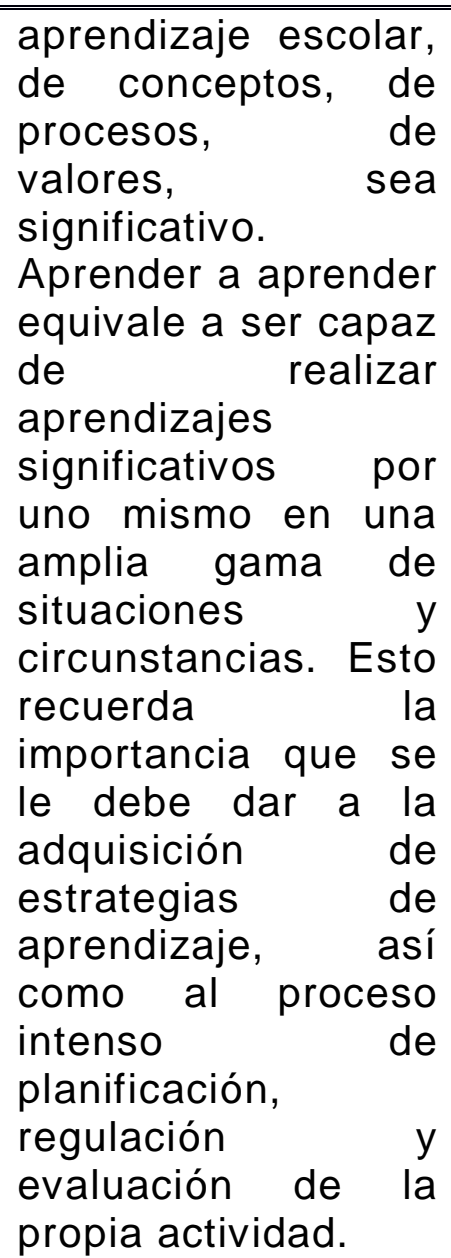 \\
\hline
\end{tabular}

Las percepciones del aprendizaje, fenómeno influyente en la formación docente de calidad Ronald José Feo Mora 


\section{Horizontalización (ejemplo de procesamiento):}

Cuadro № 3. Categoría de análisis: Aprendizaje

Subcategoría: Importancia de Aprender

\begin{tabular}{|c|c|c|}
\hline 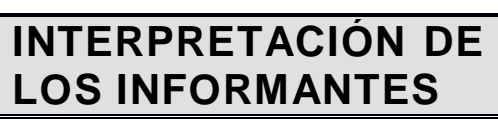 & $\begin{array}{l}\text { INTERPRETACIÓN } \\
\text { DEL INVESTIGADOR } \\
\end{array}$ & $\begin{array}{l}\text { XTO } \\
0 \\
\end{array}$ \\
\hline $\begin{array}{l}\text { La importancia del } \\
\text { aprendizaje radica en } \\
\text { que nos permite la } \\
\text { adaptación al entorno }\end{array}$ & $\begin{array}{l}\text { La importancia del } \\
\text { aprendizaje humano } \\
\text { puede ser expresada } \\
\text { en las consecuencias } \\
\text { positivas que este } \\
\text { proceso le trae a cada }\end{array}$ & $\begin{array}{lr}\text { Argyris (citado por } \\
\text { Rincón } \\
\text { Villalobos, } 2003 \text { ) } \\
\text { entiende la acción } \\
\text { como la manera que }\end{array}$ \\
\hline $\begin{array}{l}\text { Lo importante de este } \\
\text { proceso es } \\
\text { obtenemos que } \\
\text { conocimiento de } \\
\text { ya vivido }[. . .]\end{array}$ & $\begin{array}{lr}\text { persona. A través del } \\
\text { aprendizaje se procesa } \\
\text { información y } \\
\text { aprenden } \\
\text { conceptos que } \\
\text { maneian se } \\
\text { en }\end{array}$ & $\begin{array}{l}\text { la. Es la forma } \\
\text { verse a sí } \\
\text { os y a los } \\
\text { s, es a través }\end{array}$ \\
\hline $\begin{array}{l}\text { Lo complejo y varado } \\
\text { del aprendizaje } \\
\text { humano es lo que } \\
\text { provee de importancia } \\
\text { y variedad a la vida. }\end{array}$ & $\begin{array}{l}\text { contexto formal o } \\
\text { informal, lo que origina } \\
\text { una comunicación } \\
\text { entre sujetos. } \\
\text { lgualmente, por medio } \\
\text { del anrendizaie se }\end{array}$ & $\begin{array}{l}\text { crean las } \\
\text { turas } \\
\text { es, } \\
\text { se orienta a } \\
\text { uir } \\
\text { var el orden }\end{array}$ \\
\hline $\begin{array}{l}\text { reta } \\
\text { e da } \\
\text { es } \\
\text { rlo }\end{array}$ & $\begin{array}{l}\text { a expresión de } \\
\text { timientos que } \\
\text { las acciones } \\
\text { cia Otra } \\
\text { cia del }\end{array}$ & $\begin{array}{l}\text { social que se } \\
\text { requiere } \\
\text { manejar la vida. Se } \\
\text { entiende, que el } \\
\text { conocimiento para }\end{array}$ \\
\hline $\begin{array}{r}\text { se } \\
\text { de } \\
\text { itiva }\end{array}$ & $\begin{array}{l}\text { aprendizaje es que } \\
\text { auxilia al sujeto } \\
\text { mientras solventa una } \\
\text { situación de variada } \\
\text { complejidad. El mundo }\end{array}$ & $\begin{array}{l}\text { la acción es aquél } \\
\text { que utilizan los } \\
\text { actores para } \\
\text { construir su mundo, } \\
\text { estas acciones }\end{array}$ \\
\hline $\begin{array}{r}\text { es } \\
\text { ar es } \\
\text { ר uno }\end{array}$ & $\begin{array}{l}\text { humano ha } \\
\text { comprendido este } \\
\text { fenómeno a plenitud y } \\
\text { lo demuestra, por } \\
\text { ejemplo, con la } \\
\text { certificación } \\
\text { profesional, donde } \\
\text { cada sujeto debe }\end{array}$ & $\begin{array}{l}\text { estarían imbuidas } \\
\text { de significados por } \\
\text { los sujetos en su } \\
\text { interacción con el } \\
\text { mundo empírico. } \\
\text { Ello explica la } \\
\text { concordancia entre } \\
\text { acción }\end{array}$ \\
\hline
\end{tabular}

Las percepciones del aprendizaje, fenómeno influyente en la formación docente de calidad Ronald José Feo Mora 


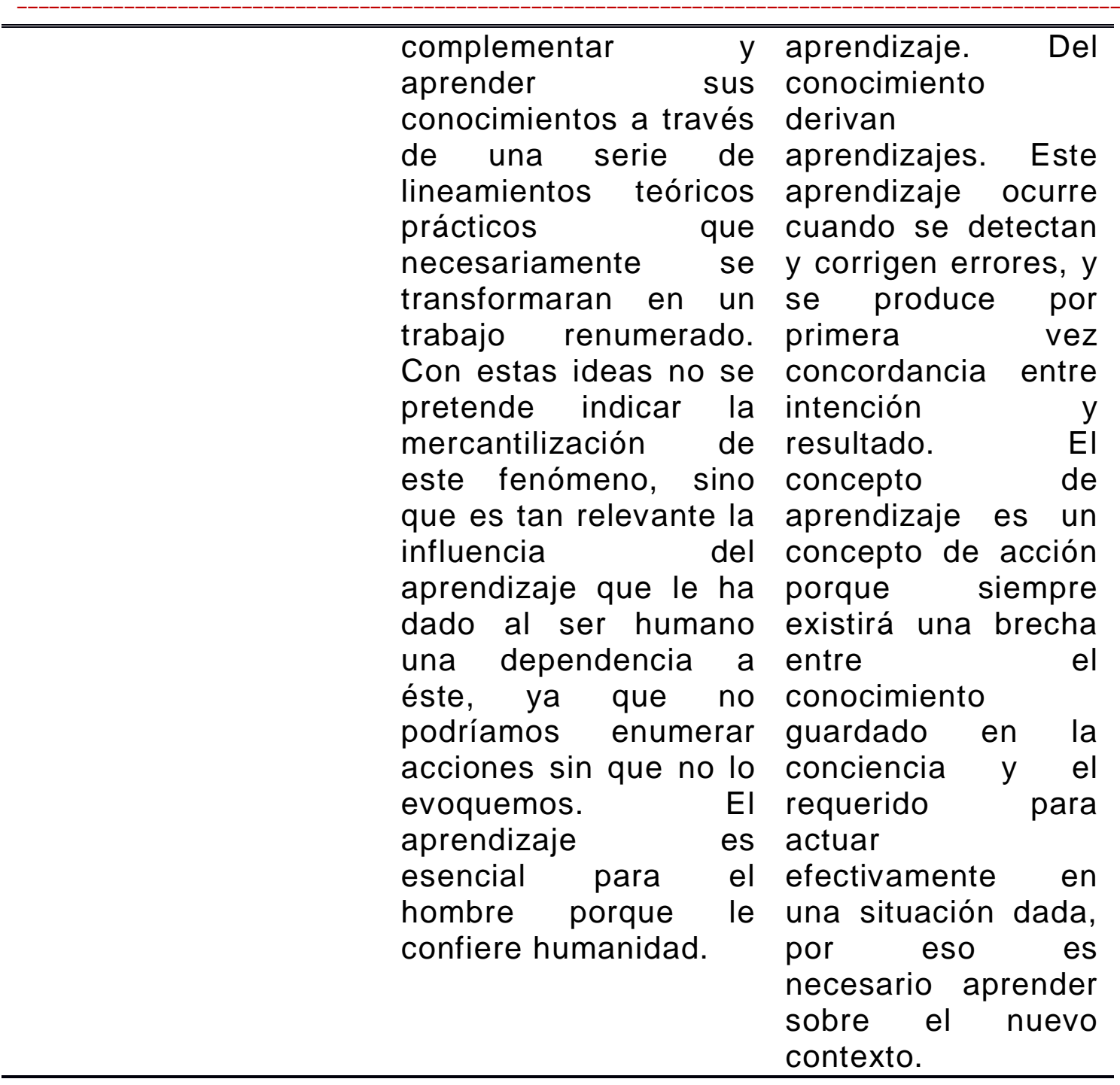

\section{Reducción Transcendental; Síntesis Conceptual:}

En a realidad en el Instituto Pedagógico de Miranda José Manuel Siso Martínez (IPMJMSM), en referencia a la percepción del aprendizaje como fenómeno influyente en la formación docente, concretamente cuando se observa o escucha al profesor y al estudiante del curso Desarrollo de Procesos Cognitivos, emergen elementos que orientan el significado de este hacia la construcción de conocimiento, lo que le da importancia como actividad humana. Esta percepción permite al sujeto valorar su relación directa con la comprensión del mundo

Las percepciones del aprendizaje, fenómeno influyente en la formación docente de calidad Ronald José Feo Mora 
donde interactúa. El aprendizaje visto como actividad humana se somete a la percepción, a las creencias, a los valores y a la historia de vida que posea quién lo defina. Esto lleva a suponer que el proceso de aprendizaje depende en gran medida de la personalidad del sujeto, y esta a su vez funge como un detonante interno de la motivación y la cognición.

Otro factor influyente en el aprendizaje son los elementos externos, constituyentes del contexto donde pertenece el individuo, como, por ejemplo: (a) el profesor; (b) el campus universitario; (c) las acciones de enseñanza; y (d) el grupo de compañeros de clase. Algo categórico sobre la percepción del aprendizaje es que dependiendo del estamento que lo define emerge una direccionalidad que lo involucra con su actor clave. El profesor lo orienta desde los medios didácticos que emplea para enseñar y el estudiante expresa las características esenciales del aprendizaje desde el punto inicial de los procedimientos que él realiza para procesar la información que le presenta el profesor.

El ser humano siempre tiene un motivo para aprender, una curiosidad interna que le lleva a la activación de procesos cognitivos y afectivos que le permiten procesar la información del exterior o recordar experiencias que lo lleven a la construcción de un conocimiento útil. EI aprendizaje inicialmente es un hecho psicológico, por eso, va de la mano a los motivos, los cuales son influenciados por la personalidad del sujeto y la representación de mundo que este posea, es decir, su yo interno. Igualmente, no se puede hablar del aprendizaje, sin reconocer la existencia de una influencia social sobre el sujeto que aprende de la interacción entre pares, de lo histórico y lo cultural. Un ser humano que contextualiza sus acciones y autorregula sus pensamientos desarrolla, o saca provecho de manera equilibrada a su yo social con otros pares.

En el ámbito de la formación docente predomina una percepción del aprendizaje vinculada de manera espontánea a términos y procesos como la adquisición de conocimiento, al dominio de habilidades y la modificación de conductas. Este fenómeno hace suponer, que el modelo formativo inserto en la ideología de los actores clave, que conforman a las

Las percepciones del aprendizaje, fenómeno influyente en la formación docente de calidad Ronald José Feo Mora 
instituciones educativas a una década del siglo XXI, sigue influenciado hacia un enfoque rígido de la educación. Este enfoque reduce el aprendizaje a un producto de las contingencias ambientales, a las conductas observables y a las respuestas del estudiante que le permiten la adquisición o el incremento de una conducta esperada y admitida por el enseñante. A la par de este enfoque surgen tendencias y acciones docentes que dan reconocimiento al aprendizaje como un proceso propio del estudiante, lo cual subyace en asideros de enfoques como el cognitivista y el constructivista.

El aprendizaje visto como adquisición de conocimientos de manera exclusiva, podría interpretarse como un concepto inexacto que repercute directamente en las acciones de la formación docente, ya que descarta que el sujeto procese información y luego bajo la influencia de sus intereses y las demandas del contexto construye conceptos potencialmente transferibles a la realidad; a este conjunto de conceptos útiles se le denominan conocimientos.

Las acciones docentes, dirigidas bajo el reconocimiento del modelo de procesamiento de la información, el apoyo y la motivación, despliegan procesos de enseñanza y aprendizaje sólidos, contextualizados a la cotidianidad humana que fortalecen la personalidad del sujeto de manera intrínseca y extrínseca, lo que podría denominarse como un potenciador para el desarrollo. Esta integración forma la personalidad del sujeto; en consecuencia, todo sujeto para desarrollarse en un contexto debe poseer un estado psicológico que le permita flexibilizar sus cogniciones, de este modo procesar la información y agruparla según sus características esenciales. En este sentido, producto de la información recolectada la categoría aprendizaje en este estudio puede ser subcategorizada en:

Aprender es un proceso humano que emerge de la interacción de elementos físicos y acciones psicológicas propias del sujeto que las activa, forjadas por la percepción sobre la realidad del contexto donde se desarrolla. Aprender posee un carácter individual, es propio de cada individuo, motorizado por los motivos con base en la interpretación de los sucesos

Las percepciones del aprendizaje, fenómeno influyente en la formación docente de calidad Ronald José Feo Mora 
que el sujeto estima adecuados, que le permiten construir un sistema de interacciones beneficiosas para él y sus pares. Estos beneficios pueden ser personales y sociales. Entre los personales se encuentra la construcción de conocimientos útiles para solucionar problemas y la concreción de la personalidad; en los sociales se delimita la consolidación de un grupo familiar y el desarrollo profesional. De esta manera, aprender se hace vital para todo ser humano, lo que significa centrar la mirada hacia una educación que se centre en el aprendizaje como elemento de progreso para la humanidad. Los modelos de formación docente deben combinar elementos naturales y cotidianos en las aulas de clases. Esta es una manera de generar sujetos de personalidad sólida y profesionales; lo cual se puede evidenciar en las siguientes expresiones:

Aprender es un proceso por medio del cual los individuos adquieren conocimiento, destrezas y habilidades que le permiten modificar conductas e inclusive valores ya presentes en su ser. (I1, 02 - 04)

Las nuevas tendencias reorientan a la persona hacia la construcción o reconstrucción acerca de lo que aprenden, rescatando así la movilidad del conocimiento; de allí que bajo esta perspectiva, lo más importante es quién aprende, entendido como un ser único, diferente, contextualizado, síntesis de sus experiencias y de las experiencias del entorno. (14, 02 - 06)

Una persona puede aprender lo que es una casa y posteriormente enriquecer este conocimiento aprendiendo que hay diferentes tipos de casas, o reestructurar este conocimiento al aprender que no todas las casas son hogares, y las habilidades y/o destrezas ya adquiridas pueden ser potenciadas $(17,11-15)$

Aprender es toda aquella nueva información, habilidades, conocimientos, actitudes que adquirimos y la modificamos mediante nuestras experiencias vividas, clasificándolas como las variantes que conocemos comúnmente o como las que no identificamos, pero le creamos un nuevo concepto para nuestro aprendizaje. (18, 02 - 06)

Las percepciones del aprendizaje, fenómeno influyente en la formación docente de calidad Ronald José Feo Mora

DOI: http://dx.doi.org/10.22458/caes.v8i1.1772

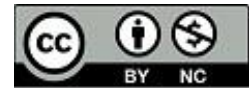

Artículo protegido por licencia Creative Commons 
Aprendemos por necesidad, desde que nacemos y a lo largo de toda nuestra existencia estamos en ese constante proceso de aprendizaje. (I10, 08 - 10)

Es evidente que aprender es un proceso humano que se ha vinculado con asuntos formales de la educación, esto permitió concebir representaciones científicas para comprenderlo; esta orientación se extendió hacia modelos de formación docente que lo tecnificaban y en consecuencia lo deshumanizaban. Consecutivamente, se generó un mayor obstáculo, el cual radicó en la creencia indiscutible, que el mejor conocimiento es aquel que ha sido comprobado bajo unos estándares concertados por un grupo de estudiosos o modelo económico predominante. Esta tendencia aun predominante en los sistemas educativos formales, cuestiona que aprender es un proceso psicológico y no en exclusiva científico, que por naturaleza responde desde lo experimental, representado en las formas de intercambio de experiencias de un sujeto y su contexto de desarrollo. La escuela debe girar sus acciones hacia la proyección de situaciones locales y comunes hasta construir proposiciones útiles para el estudiante, y no para una sociedad de intereses con orientaciones económicas y científicas.

La importancia de aprender reside en su correspondencia con el desarrollo humano, ya que aprender provee al sujeto de información útil sobre su entorno para generar metas personales y sociales; de esta manera, todo individuo que aprenda de manera consciente que valora sus conocimientos extiende las posibilidades de progreso en un mundo lleno de constante cambios y exigencias. De ahí que el sistema de formación docente está llamado a incorporar esta manifestación en todos y cada uno sus espacios cotidianos y académicos. El estudiante que sea educado con una concepción clara y consolidada sobre lo importante de estimar lo que aprende, será un profesional de la docencia que exteriorice a sus condiscípulos, en cada encuentro pedagógico, el valor de asimilar y construir conocimientos a partir de la discusión de saberes; en consecuencia, si un estudiante puede valorar lo aprendido desde la visión que se opone a la idea de estudiar solo como un requisito para

Las percepciones del aprendizaje, fenómeno influyente en la formación docente de calidad Ronald José Feo Mora

DOI: http://dx.doi.org/10.22458/caes.v8i1.1772

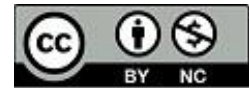

Artículo protegido por licencia Creative Commons 
aprobar la materia, más bien para tomar conciencia sobre los conocimientos significativos alcanzados, le será sencillo transferir estos a escenarios exigentes de competencias específicas que le ayuden a solventar situaciones problemáticas.

Inevitablemente la importancia de aprender lleva de la mano al sujeto a comprender y a reconocer la necesidad de estudiar y actualizarse continuamente, esto último equivale, a que el individuo reconozca al aprendizaje como un factor directamente relacionado a su personalidad y a sus motivos para aprender. Ejemplo de ello, son los indicadores que se citan a continuación:

Es importante, ya que es lo que permite que el ser humano crezca, se desarrolle, social, profesional e intelectualmente. (I1, 08 - 09)

Permite ampliar el potencial de conocimientos de cada individuo, el desarrollo de sus competencias cognitivas, la comprensión de tus propios pensamientos bajo la reflexión, logrando que la persona tome conciencia de sus propios pensamientos para el logro de la resolución de problemas y la toma de decisiones. (I3, 10 - 14)

Es entonces que el aprender juega un papel muy importante, ya que a través de los diferentes procesos de aprendizajes se adquieren, potencian y refinan los conocimientos, habilidades y destrezas para la supervivencia de la especie. (17, $25-28)$

Es de gran importancia, porque con ello es que el ser humano se podrá defender eficaz y moralmente en el transcurso de su vida. (I8, 08 - 09)

El aprendizaje es sumamente importante, porque debido a ese proceso moldeamos nuestra conducta, desarrollamos habilidades y destrezas, nos formamos moralmente, llegamos a ser útiles y productivos y logramos además una realización personal. $(110,15-18)$

Las percepciones del aprendizaje, fenómeno influyente en la formación docente de calidad Ronald José Feo Mora

DOI: http://dx.doi.org/10.22458/caes.v8i1.1772

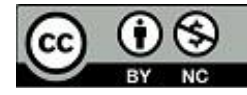


Existen cuatro variables fundamentales que influyen en el aprendizaje de los seres humanos: (a) la percepción de la realidad; (b) el contexto histórico - cultural, donde habita el sujeto; (c) la personalidad del sujeto; y (e) las relaciones con sus pares. Un estudiante en formación docente necesariamente debe conocer estas variables para que, en primer lugar, comprenda como son sus procesos y acciones para aprender, para luego generar estrategias que ayuden e involucren a sus futuros aprendices a dilucidar como ellos aprenden. Este es el camino que invita y que construye la nueva era escolar, donde se valora al aprendizaje como vínculo que nos une a nuestros ancestros en la imperiosa necesidad de coexistir con los pares y el propio contexto. Este cambio de percepción es una línea de acción que fortalece la formación docente, ya que le concede al estudiante la toma de conciencia sobre la importancia de aprender.

\section{REFLEXIONES CONCLUSIVAS}

1.- Los sistemas de formación docente de calidad se encuentran alejados de las expresiones y experiencias que reducen el aprendizaje como adquisición de conocimiento, dominio de habilidades y modificación de conductas. Sin embargo, lo real es la tendencia de los modelos predominantes insertos en los sistemas de formación docente se encuentran aún bajo la influencia de enfoques reduccionistas sobre el aprendizaje, lo que genera un sistema educativo centrado en la exposición de saberes disciplinares. Esta situación genera dos consecuencias, vinculadas entre sí que nos aleja de una propuesta óptima de formación de calidad. La primera, se refiere a la insuficiencia de la promoción de estrategias de aprendizaje por parte del docente para la comprensión del contenido escolar por parte del estudiante; la segunda, trae consigo que el estudiante en formación docente posee la tendencia de mecanizar los procesos cognitivos y las actividades para solventar una situación problemática, que asume las dificultades al aprender como un hecho natural y que delega al docente la iniciativa y las acciones para aprender. Estos acontecimientos favorecen la idea en el estudiante, que el aprendizaje es un hecho emanado del subconsciente, de la memorización sin significado y del acopio de información para aprobar

Las percepciones del aprendizaje, fenómeno influyente en la formación docente de calidad Ronald José Feo Mora 
una asignación escolar; además, de pensar que el aprendizaje es un proceso que se encuentra por encima de la toma de conciencia de las acciones pertinentes para asimilar una información.

2.- Los hallazgos emergentes reflejan elementos propios de una definición del aprendizaje en los estamentos docente y estudiante como construcción del conocimiento, lo que le da categoría de actividad humana. Esto permite comprender y valorar el aprendizaje con su relación directa del mundo donde interactúa el sujeto. Dichas representaciones someten el aprendizaje a la percepción, a las creencias, a los valores y a la historia de vida de cada individuo, lo que admite presumir que el aprendizaje se soslaya a la personalidad del sujeto como un detonante interno de la motivación y la cognición. Los sistemas de formación docente se encuentran llamados a interpretar esta aserción para diseñar acciones que fomenten una visión amplia sobre las formas de aprender, desde la concepción académica hasta el propio ser. Es quizás la única forma de establecer un complejo sistema de formación docente de calidad que gire en torno a la humanidad, la gestión del saber y la armonía del entorno donde se desarrolla el sujeto.

3.- Algo particular sobre la percepción del aprendizaje en la formación docente de calidad es que dependiendo del estamento emergen características esenciales particulares que lo definen. Los profesores y estudiantes perciben que los elementos influyentes sobre el aprendizaje son: (a) profesor; (b) campus universitario; (c) acciones de enseñanza; y (d) grupo de compañeros de clase. Esta situación reafirma la complejidad de los sistemas de formación docente de calidad ya que, al ser guiados por un currículo preestablecido, bien sea por expertos o construido desde la apertura de las bases, siempre existirá una tendencia demarcada por la percepción del aprendizaje que enriquece al propio sistema y lo hace de manera particular, ya que en esencia responde a la necesidad humana de innovación.

4.-El aprendizaje en la formación docente de calidad es determinado por una dinámica compleja de procesos cognitivos que van más allá de las estrategias de adquisición, almacenaje y recuperación de información, estos procedimientos pueden ser regulados y

Las percepciones del aprendizaje, fenómeno influyente en la formación docente de calidad Ronald José Feo Mora

DOI: http://dx.doi.org/10.22458/caes.v8i1.1772

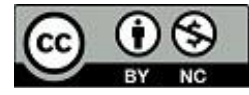

Artículo protegido por licencia Creative Commons 
controlados bajo el apoyo de procesos cognitivos superiores como la metacognición en compañía de un motivo que origina la construcción deliberada de estrategias de aprendizaje, lo que hace al sujeto arquitecto de su propio conocimiento.

\section{REFERENCIAS}

Abbagnano, N. (1986). Diccionario de Filosofía. México: Fondo de Cultura Económica.

De Lella, C. (1999). Modelos y Tendencias de la Formación Docente. Ponencia presentada en el Seminario sobre estrategias de Formación. Perú: OEl.

Domínguez, M. (2010). Hacia una Didáctica de la Composición Escrita Estratégica. Trabajo de Ascenso no publicado, Universidad Pedagógica Experimental Libertador, Instituto Pedagógico de Miranda José Manuel Siso Martínez, Caracas.

Hernández, G. (1998). Paradigmas en Psicología de la Educación. México: Paidos.

Husserl, E. (1949). Ideas Relativas a una Fenomenología Pura y una Filosofía Fenomenológica. México: FCE.

ICH. (2010). Instituto de Ciencias del Hombre. [Página Web en línea]. Recuperado de: http://www.ich.es. [Consulta: 2016, diciembre 04].

Moya, J. (2007). Conferencia Qué Podemos Entender por Competencias. [Video en línea]. Recuperado de http: //procesos.webnode.com. [Consulta: 2010, julio, 15].

Ponce, S., Lomeli, C., Roa, R. Martínez y Oviedo, E. (2010). Resultados de una Propuesta de Formación para Docentes Principiantes de Educación Superior. [Documento en línea] Ponencia presentada en el Segundo Congreso Internacional sobre Profesorado Principiante e Inserción Profesional a la Docencia. Acompañamiento a los docentes noveles: prácticas y concepciones. Recuperado de http://cedoc.infd.edu.ar/noveles/principiantes [Consulta: 2016, junio 28].

Pozo, J. (1999). Aprendices y Maestros. La Nueva Cultura del Aprendizaje. Madrid: Alianza Editorial.

Sánchez, V. y Jurado, F. (2000). La Práctica como Motor de la Transformación Docente (Inicial y Continua). Colombia: Cooperativa Magisterio.

Las percepciones del aprendizaje, fenómeno influyente en la formación docente de calidad Ronald José Feo Mora

DOI: http://dx.doi.org/10.22458/caes.v8i1.1772

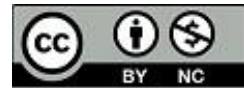


SESM (2010). Subsecretaria de Educación Superior. [Página Web en línea]. Recuperado de http://ses.sep.gob.mx. [Consulta: 2016, diciembre 04].

Taylor, R. y Bogdan, T. (1987). Introducción a los Métodos Cualitativos de Investigación. México: Paidos.

Tejada, F. (2000). Perfil Docente y Modelo de Formación. México: Fondo Iberoamericano sobre Educación en Valores.

Téllez, M. Rodríguez, N., Lacueva, A., Córdova, D., Gracía, J., Amaro, R. y Sayago, Z. (2009). Los Retos de la Formación Docente. Caracas: Lab. Educativo.

Vargas, L. (1994). Sobre el Concepto de Percepción. Alteridades 4(8), 47- 53.

Las percepciones del aprendizaje, fenómeno influyente en la formación docente de calidad Ronald José Feo Mora

DOI: http://dx.doi.org/10.22458/caes.v8i1.1772

\section{(c) (1) $(9)$}

